\title{
Article
}

\section{Effects of $\mathrm{Bi}$ and $\mathrm{Ni}$ on the properties of a vanadium phosphorus oxide catalyst}

\author{
Yun Hin Taufiq-Yapa,b,*, Choon Seon Yuen a,b, Nawi @ Mohamed Nurul Suziana ${ }^{\mathrm{a}, \mathrm{b}}$, \\ Ramli Irmawati a,b \\ a Catalysis Science and Technology Research Centre, Faculty of Science, University Putra Malaysia, 43400 UPM Serdang, Selangor, Malaysia \\ b Department of Chemistry, Faculty of Science, Universiti Putra Malaysia, 43400 UPM Serdang, Selangor, Malaysia
}

\section{A R T I C L E I N F O}

\section{Article history:}

Received 5 August 2013

Accepted 14 November 2013

Published 20 February 2014

\section{Keywords:}

Vanadium phosphorus oxide catalyst

Promoter

$n$-Butane oxidation

Maleic anhydride selectivity

\begin{abstract}
A B S T R A C T
Vanadium phosphorus oxide (VPO) catalysts were synthesized by the dihydrate method which involved the two steps for the preparation of the dihydrate $\left(\mathrm{VOPO}_{4} \cdot 2 \mathrm{H}_{2} \mathrm{O}\right)$ and the precursor hemihydrate $\left(\mathrm{VOHPO}_{4} \cdot 0.5 \mathrm{H}_{2} \mathrm{O}\right)$. $\mathrm{Bi}$ and $\mathrm{Ni}$ salt were added into the mixture of $\mathrm{VOPO}_{4} \cdot 2 \mathrm{H}_{2} \mathrm{O}$ and isobutanol, and the obtained precursors were calcined in a flow of a $n$-butane/air mixture to produce the promoted VPO catalysts. The catalysts were characterized by X-ray diffraction (XRD), $\mathrm{N}_{2}$ adsorption-desorption, inductively coupled plasma-atomic emission spectroscopy, scanning electron microscopy (SEM), and $\mathrm{H}_{2}$ temperature-programmed reduction $\left(\mathrm{H}_{2}\right.$-TPR). Their catalytic properties were tested using a fixed-bed microreactor. All the catalysts gave main XRD peaks at $2 \theta=22.9^{\circ}$, $28.5^{\circ}$, and $30.0^{\circ}$, attributing to the (020), (204), and (221) planes of the pyrophosphate phase (VO) ${ }_{2} \mathrm{P}_{2} \mathrm{O}_{7}$, respectively. The promoted catalysts have smaller crystallite size and higher specific surface areas. SEM micrographs revealed the formation of more prominent plate-like crystallites that were arranged as rosette clusters. $\mathrm{H}_{2}$-TPR results showed that the promoted catalysts had lower reduction peak temperatures and possessed higher amounts of $\mathrm{V}^{5+}-\mathrm{O}^{2-}$ and $\mathrm{V}^{4+-} \mathrm{O}^{-}$pairs, which gave higher selectivity and activity in the selective oxidation of $n$-butane to maleic anhydride.

(C) 2014, Dalian Institute of Chemical Physics, Chinese Academy of Sciences. Published by Elsevier B.V. All rights reserved.
\end{abstract}

\section{Introduction}

Maleic anhydride (MA) is an important petrochemical and polymer feedstock [1]. It is produced by the selective oxidation of $n$-butane over vanadium phosphorus oxide (VPO) catalysts, which is better than the use of benzene because butane is less toxic, less expensive and no carbon is lost in the oxidation [2]. VPO catalyst is well known as the active phase and is commercially used [3], which has been extensively studied. To date it is the only industrial commercial catalyst for the selective oxidation of alkane [4].

The introduction of a metal promoter into the VPO catalyst is an effective method for improving its catalytic properties [5]. The promoter plays an important role in the adsorption of oxygen and its diffusion in the lattice by which a nonselective route of $n$-butane oxidation is suppressed [6]. According to Centi et al. [7], the effects of promoters can be divided into three categories as follows. Category 1 is the addition of ions that interact with free phosphoric acid as a means to fine-tune the optimum surface P/V ratio and acidity. Category 2 is the addition of ions that substitute for $\mathrm{P}$ in the precursor. The partial or total elimination of these ions from the vanadyl pyrophosphate structure by calcination influences the morphology and leads to defects in the $(\mathrm{VO})_{2} \mathrm{P}_{2} \mathrm{O}_{7}$ structure. Category 3 is

\footnotetext{
* Corresponding author. Tel: +60-3-89466809; Fax: +60-3-89466758; E-mail: yap@science.upm.edu.my This work was supported by the Ministry of Science, Technology and Innovation of Malaysia. DOI: 10.1016/S1872-2067(12)60749-9 | http://www.sciencedirect.com/science/journal/18722067 | Chin. J. Catal., Vol. 35, No. 2, February 2014
} 
the addition of elements that substitute for $\mathrm{V}$ and act as modifiers of the activity by forming stable solid solutions.

Among the most investigated promoters for VPO catalysts, Bi has been used for the past two decades [8-11], and high conversion (71\%) and selectivity (68\%) have been reported for $1 \%$ Bi promoted catalysts [12]. Ni increases the mobility of the lattice oxygen and lowers the temperature of the reduction peaks $[13,14]$. High conversion $(87.2 \%)$ and selectivity $(57.5 \%)$ have been obtained for $1 \% \mathrm{Ni}$ promoted catalysts [5]. The role of Bi-Ni bimetallic promoters on the physicochemical and catalytic properties of VPO catalysts for $n$-butane oxidation was studied here.

\section{Experimental}

\subsection{Catalyst preparation}

In first step, the dihydrate, $\mathrm{VOPO}_{4} \cdot 2 \mathrm{H}_{2} \mathrm{O}$, was prepared by reacting $\mathrm{V}_{2} \mathrm{O}_{5}\left(60.0 \mathrm{~g}\right.$, Fluka) with aqueous $o-\mathrm{H}_{3} \mathrm{PO}_{4}$ (577.5 g, $85 \%$, J.T. Baker) in distilled water ( $24 \mathrm{~mL} / \mathrm{g}$ solid). The mixture was then refluxed with continuous stirring for $24 \mathrm{~h}$. The yellow solid product was recovered by centrifuging, washed with acetone and distilled water, and dried at $373 \mathrm{~K}$ for $24 \mathrm{~h}$ in an oven.

In the second step, $1 \%$ or $2 \%$ (molar faction) of $\mathrm{Bi}$ and $\mathrm{Ni}$ salts $\left(\mathrm{Bi}\left(\mathrm{NO}_{3}\right)_{3} \cdot 5 \mathrm{H}_{2} \mathrm{O}\right.$ and $\left.\mathrm{NiSO}_{4} \cdot 6 \mathrm{H}_{2} \mathrm{O}\right)$ were first dissolved in $160 \mathrm{ml}$ isobutanol (99.9\%, Fisher Chemical) before they were added into a reaction mixture with $8 \mathrm{~g} \mathrm{VOPO} \cdot 2 \mathrm{H}_{2} \mathrm{O}$ to prepare the promoted precursor hemihydrate, $\mathrm{VOHPO}_{4} \cdot 0.5 \mathrm{H}_{2} \mathrm{O}$. The mixture was rapidly stirred and refluxed for $21 \mathrm{~h}$. The blue solid product was removed by centrifuging, washed with distilled water and acetone, and dried at $373 \mathrm{~K}$ for $24 \mathrm{~h}$ in an oven.

Finally, the unpromoted and promoted precursors, VOHPO ${ }_{4} \cdot 0.5 \mathrm{H}_{2} \mathrm{O}$, were calcined at $733 \mathrm{~K}$ in a flow of $0.75 \%$ $n$-butane/air mixture for $18 \mathrm{~h}$. The unpromoted catalyst was denoted as CatUnpromoted; the promoted catalysts with $\mathrm{Bi} / \mathrm{Ni}$ $=0.5,1$, and 2 were denoted as CatBi1Ni1, CatBi1Ni2, and CatBi2Ni1, respectively.

\subsection{Catalysts characterization}

X-ray diffraction (XRD) patterns of the catalysts at ambient temperature were obtained by a Shimadzu Diffractometer (Model XRD-6000) employing $\mathrm{Cu} K_{\alpha}$ radiation. The BET (Brunauer-Emmett-Teller) method was used to measure the total specific surface areas of the VPO catalysts using a Thermo Finnigan Sorptomatic 1990 nitrogen adsorption-desorption analyzer at $77 \mathrm{~K}$. The elemental compositions of the VPO catalysts were determined by a Perkin Elmer emission spectrometer (Model Plasma 1000) and sequential scanning inductively coupled plasma-atomic emission spectroscopy (ICP-AES). Scanning electron microscopy (SEM) was performed using a Leo 1455 VP electron microscope. The samples were coated with gold using a Sputter Coater. Temperature-programmed reduction ( $\mathrm{H}_{2}$-TPR) analysis was performed using a Thermo Finnigan TPDRO 1110 apparatus with a thermal conductivity detector. The program used to determine the amount of oxygen atoms was TPD/R/O 1100 version 2.3 .

\subsection{Catalytic test}

A standard mass of catalyst $(0.25 \mathrm{~g})$ was used for the oxidation of $n$-butane to MA in a fixed-bed microreactor at $673 \mathrm{~K}$ with a gas hourly space velocity (GHSV) of $2400 \mathrm{~h}^{-1}$. $n$-Butane and air were fed into the reactor by calibrated mass flow controllers to give a feedstock composition of $1 \% n$-butane in air. The products were fed by heated lines to an online gas chromatography for product analysis. The reactor comprised a stainless steel tube with the catalyst held in place by plugs of quartz wool. A thermocouple was located in the centre of the catalyst bed and the temperature control was typically $\pm 5 \mathrm{~K}$.

\section{Results and discussion}

\subsection{XRD results}

Figure 1 shows the XRD patterns of the unpromoted and promoted precursors, which perfectly matched the standard reference (JCPDS File No. 37-0269) of the hemihydrate, $\mathrm{VOHPO}_{4} \cdot 0.5 \mathrm{H}_{2} \mathrm{O}$. The main peaks at $2 \theta=15.5^{\circ}, 19.6^{\circ}, 24.1^{\circ}$, $27.0^{\circ}$, and $30.3^{\circ}$ corresponded to the (001), (101), (021), (121), and (130) planes, respectively. These confirmed that all the precursors have $\mathrm{VOHPO}_{4} \cdot 0.5 \mathrm{H}_{2} \mathrm{O}$ phase. The promoted precursors did not show any extra peak in the XRD pattern. They had decreased crystallinity as compared to the unpromoted hemihydrates, which was reflected by the lower intensity of the peaks. According to Haber et al. [15], the (001) plane of VOHPO $_{4} \cdot 0.5 \mathrm{H}_{2} \mathrm{O}$ phase is responsible for the catalytic activity in the selective oxidation of $n$-butane and it is transformed into the topotactically similar $(020)$ plane of $(\mathrm{VO})_{2} \mathrm{P}_{2} \mathrm{O}_{7}$.

In Fig. 2, the XRD patterns of all the promoted VPO catalysts showed the same characteristic peaks for the (VO) ${ }_{2} \mathrm{P}_{2} \mathrm{O}_{7}$ phase as the unpromoted one (JCPDS File No. 34-1381). The characteristic peaks of these VPO catalysts appeared at $2 \theta=22.9^{\circ}$, $28.5^{\circ}$, and $30.0^{\circ}$, which corresponded to the (020), (204), and (221) planes, respectively.

The crystallite size of the unpromoted and promoted VPO catalysts was calculated using the Debye-Scherrer equation [16] and the width of the peaks of the (020) and (204) planes,

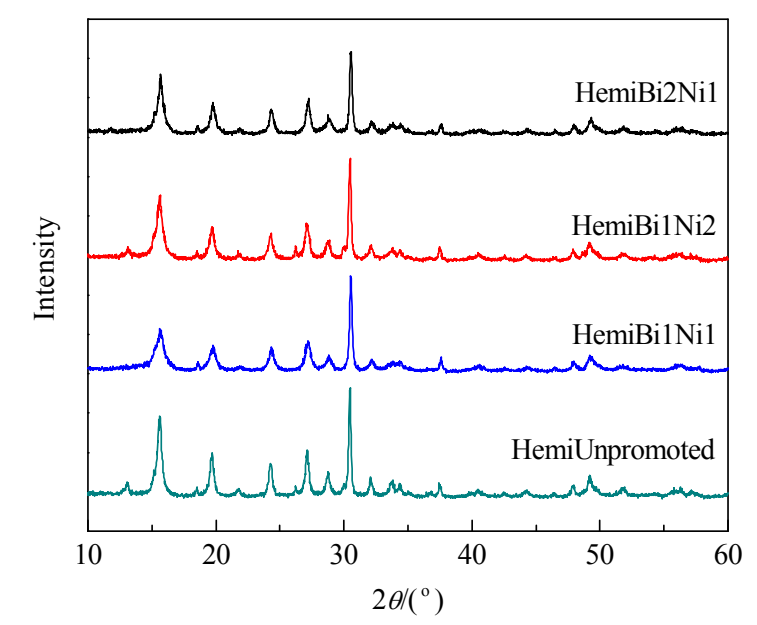

Fig. 1. XRD patterns of the precursor hemihydrate, $\mathrm{VOHPO}_{4} \cdot 0.5 \mathrm{H}_{2} \mathrm{O}$. 


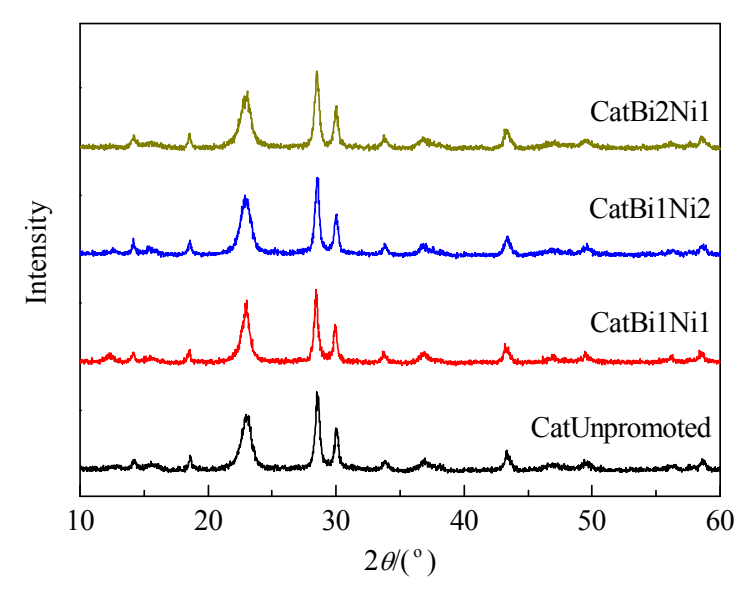

Fig. 2. XRD patterns of unpromoted and promoted VPO catalysts.

and the results are summarised in Table 1 . All the promoted VPO catalysts have smaller size as compared with the unpromoted catalyst, which was $134.73 \AA$ from the (020) plane and $276.57 \AA$ from the (204) plane. Among the promoted VPO catalysts, CatBi1Ni1 was the smallest in size, 83.54 $197.02 \AA$ from the (020) and (204) planes, respectively. The crystallite sizes of CatBi1Ni2 and CatBi2Ni1 from the (020) plane were 84.62 and $85.81 \AA$, respectively, while the crystallite size of the same series of catalysts from the (204) plane were 197.33 and $208.36 \AA$, respectively. It can be thus concluded that even though there was no phase change detected, the addition of $\mathrm{Bi}$ and Ni promoters had affected the microstructure of the synthesized VPO catalyst. The increase of the full width at half maximum (FWHM) of the (020) plane diffraction indicated that the crystallite size in the (020) plane direction had decreased. The crystallite size at the (204) plane is only indicative of the mean "length", while that at the (020) plane is more representative of the actual thickness [17]. The surface which is selective for the formation of MA is the (020) plane, and side faces such as the (001) plane are active for nonselective oxidation [18].

The original VPO catalyst consists mainly of the vanadyl pyrophosphate phase, $(\mathrm{VO})_{2} \mathrm{P}_{2} \mathrm{O}_{7}$. In this study, $\mathrm{Bi}$ and $\mathrm{Ni}$ promoters were added in very small amounts $(1 \%$ or $2 \%)$. According to de Farias et al. [19], the amount of promoter added was too low to have a structural effect, which was in agreement with the XRD results. The authors proposed that the cation or promoter was incorporated into the vanadyl pyrophosphate to form solid solution of the type ( $\left.(V O)_{1-x} \mathrm{M}_{x}\right)_{2} \mathrm{P}_{2} \mathrm{O}_{7}$ where $\mathrm{M}$ represents a promoter cation. This may form defects in the structure, which can act as new active sites or, alternatively, the metal cations can affect the redox properties of the surface.

Table 1

XRD data of the VPO catalysts.

\begin{tabular}{lccccc}
\hline \multirow{2}{*}{ Catalyst } & \multicolumn{2}{c}{ Line width $\left(^{\circ}\right)$} & & \multicolumn{2}{c}{ Crystallite size $(\AA)$} \\
\cline { 2 - 3 } \cline { 5 - 6 } & $(020)$ & $(204)$ & & $(020)$ & $(204)$ \\
\hline CatUnpromoted & 0.5955 & 0.2933 & & 134.73 & 276.57 \\
CatBi1Ni1 & 0.9600 & 0.4117 & & 83.54 & 197.02 \\
CatBi1Ni2 & 0.9480 & 0.4111 & & 84.62 & 197.33 \\
CatBi2Ni1 & 0.9350 & 0.3893 & & 85.81 & 208.36 \\
\hline
\end{tabular}

Ye et al. [20] reported that the (020) plane of $(\mathrm{VO})_{2} \mathrm{P}_{2} \mathrm{O}_{7}$ was active for the selective oxidation of $n$-butane, which leads to the expectation that a high exposure of the (020) plane will result in higher activity and selectivity, but that this is not the sole factor governing catalytic performance. The authors found broadening of the (020) peak and disorder along the (020) plane after modification by the addition of promoters. On the (020) plane of $(\mathrm{VO})_{2} \mathrm{P}_{2} \mathrm{O}_{7}$, there are five kinds of oxygen sites: $\mathrm{O} . . . \mathrm{V}, \mathrm{V} 2-\mathrm{O}-\mathrm{P}, \mathrm{V}-\mathrm{O}-\mathrm{P}, \mathrm{P}-\mathrm{O}-\mathrm{P}$, and $\mathrm{V}=\mathrm{O}\left(\mathrm{V}^{5+}=\mathrm{O}\right)$. The $\mathrm{V}=\mathrm{O}$ species is expected to act as active lattice oxygen, although several researchers claimed that adsorbed oxygen species were responsible for the reaction. Since surface $\mathrm{V}=\mathrm{O}$ species are present on the (020) plane, one would expect that the surface density of $\mathrm{V}=0$ species would increase with the exposure of the (020) plane and disorder along the $(020)$ plane.

\subsection{Specific surface area measurements}

The BET surface areas of the unpromoted and promoted VPO catalysts are summarized in Table 2 . It showed that the BET surface area of the unpromoted VPO catalyst was $23 \mathrm{~m}^{2} / \mathrm{g}$. CatBi1Ni1 and CatBi1Ni2 samples have higher specific surface areas, which were 31 and $28 \mathrm{~m}^{2} / \mathrm{g}$, respectively. This indicated that the addition of $\mathrm{Bi}$ and $\mathrm{Ni}$ facilitated the formation of crystal phases with a higher surface area. These results were consistent with the XRD results (Table 1), which showed that the promoted VPO catalysts have smaller particle sizes compared with the unpromoted one.

Generally, the addition of Bi and Ni into the VPO catalyst matrix gave remarkably higher surface areas $[11,12]$. In addition, Taufiq-Yap et al. [12] found that promotion by Bi into the VPO catalyst altered the development of the basal (100) (VO) ${ }_{2} \mathrm{P}_{2} \mathrm{O}_{7}$ phase, which is an interesting feature of the high surface area of the catalysts.

Ye et al. [20] suggested that the increase of the surface area was accompanied by an increase in the (020) plane exposure of (VO) ${ }_{2} \mathrm{P}_{2} \mathrm{O}_{7}$. They also suggested that the promoter metal cations were incorporated into the interlayer spacing to reduce the weak $\mathrm{H}$ bonding of phosphate and water in $\mathrm{VOHPO}_{4} \cdot 0.5 \mathrm{H}_{2} \mathrm{O}$. As a result, cleavage along the (001) plane of the $\mathrm{VOHPO}_{4} \cdot 0.5 \mathrm{H}_{2} \mathrm{O}$ was easier, which would lead to the increase of both the surface area and exposure of the (020) plane of $(\mathrm{VO})_{2} \mathrm{P}_{2} \mathrm{O}_{7}$. According to Hutchings [21], a particular advantage of the higher surface area of the VPO catalysts is that they performed well at lower temperatures, which led to enhanced selectivity and hence high yields of product obtained.

We suggest that the added $\mathrm{Bi}$ and Ni promoters were incorporated into the interlayer spacing of $\mathrm{VOHPO}_{4} \cdot 0.5 \mathrm{H}_{2} \mathrm{O}$ to weaken the $\mathrm{H}$ bonding. Consequently, the (001) plane of the $\mathrm{VOHPO}_{4} \cdot 0.5 \mathrm{H}_{2} \mathrm{O}$ became easier to cleave and led to the increase

Table 2

BET surface areas of unpromoted and promoted VPO catalysts.

\begin{tabular}{lc}
\hline Catalyst & Surface area $\left(\mathrm{m}^{2} / \mathrm{g}\right)$ \\
\hline CatUnpromoted & 23 \\
CatBi1Ni1 & 31 \\
CatBi1Ni2 & 28 \\
CatBi2Ni1 & 27 \\
\hline
\end{tabular}


Table 3

$\mathrm{P} / \mathrm{V}$ ratio for unpromoted, Bi-promoted, and Ni-promoted VPO Catalysts.

\begin{tabular}{lccc}
\hline \multirow{2}{*}{ Catalyst } & \multicolumn{3}{c}{ Molar ratio (ICP) } \\
\cline { 2 - 4 } & $\mathrm{P} / \mathrm{V}$ & $\mathrm{Bi} / \mathrm{V}$ & $\mathrm{Ni} / \mathrm{V}$ \\
\hline CatUnpromoted & 1.04 & - & - \\
CatBi1Ni1 & 1.09 & 0.009 & 0.009 \\
CatBi1Ni2 & 1.06 & 0.008 & 0.018 \\
CatBi2Ni1 & 1.05 & 0.018 & 0.009 \\
\hline
\end{tabular}

of both the surface area and the exposure of the (020) plane of $(\mathrm{VO})_{2} \mathrm{P}_{2} \mathrm{O}_{7}$.

\subsection{Elemental analysis}

The ICP-AES results are shown in Table 3 for the $\mathrm{P} / \mathrm{V}$ ratios of the promoted VPO catalysts. These P/V values fall in the range of 1.0-1.2 required to produce the so-called active and selective (VO) ${ }_{2} \mathrm{P}_{2} \mathrm{O}_{7}$ phase [22-24]. This is good agreement with the literature that the $\mathrm{V}^{4+}$ species responsible for the catalyst activity is stabilised by a higher $\mathrm{P} / \mathrm{V}$ atomic ratio [25].

The analysis also confirmed the presence of the $\mathrm{Bi}$ and $\mathrm{Ni}$ promoter in the VPO catalysts with $\mathrm{Bi} / \mathrm{V}$ and $\mathrm{Ni} / \mathrm{V}$ atomic ratios between 0.010 and 0.020 . It can be interpreted that the introduction of different types of metal promoters at different $\mathrm{M} / \mathrm{V}$ (metal promoter/vanadium) ratios into (VO) ${ }_{2} \mathrm{P}_{2} \mathrm{O}_{7}$ have an effect on the formation of different crystal plates with different surface morphology. Therefore, we suggest that the different metal cations play different roles in the bulk system of the (VO) ${ }_{2} \mathrm{P}_{2} \mathrm{O}_{7}$ catalysts, which can significantly affect the surface area (Table 2) of the catalysts.

\subsection{SEM results}

Figure 3 shows SEM images of the unpromoted and promoted VPO catalysts. The principle structure of the catalysts was the same, and consisted of rosette-shape plate-like crystals. These crystals are made up of agglomerates of $(\mathrm{VO})_{2} \mathrm{P}_{2} \mathrm{O}_{7}$ platelets that preferentially expose the (100) crystal planes [26].
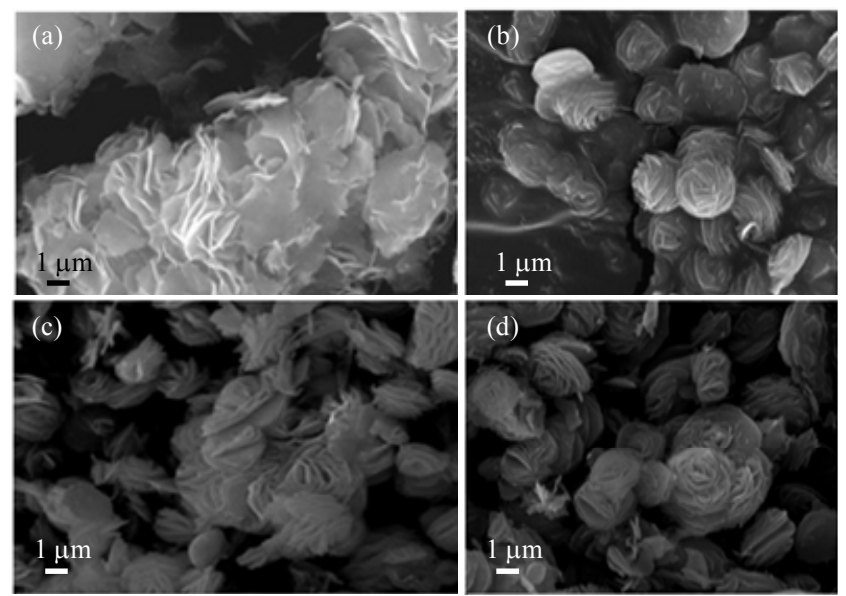

Fig. 3. SEM micrographs of unpromoted and promoted VPO catalysts. (a) CatUnpromoted; (b) CatBi1Ni1; (c) CatBi1Ni2; (d) CatBi2Ni1.
Bi and Ni promoted VPO catalysts showed a more compact structure with more layered plate-like crystals which formed at the surface of the clusters. The rosette-shape clusters observed were smaller than those of the unpromoted counterpart. This is consistent with that the promoted VPO catalysts showed higher surface areas as compared with unpromoted one.

\section{5. $\mathrm{H}_{2}$-TPR results}

Figure 4 shows the TPR profiles of the unpromoted and promoted VPO catalysts, which showed the nature of the surface and reducibility of the catalysts. All the catalysts had two reduction peaks, which were assigned to the reduction of $\mathrm{V}^{5+}$ species and the removal of lattice oxygen from $\mathrm{V}^{4+}$ species, respectively [27]. The peak attributed to $\mathrm{V}^{5+}$ is associated with the removal of $\mathrm{O}^{2-}$ anions and the peak from $\mathrm{V}^{4+}$ is related to the $0^{-}$anion oxygen species [28]. There are good relationships between the amount of oxygen species removed from the $\mathrm{V}^{5+}$ and $\mathrm{V}^{4+}$ species with MA selectivity and $n$-butane conversion, respectively [29].

As shown in Fig. 4, CatUnpromoted sample gave a shoulder first reduction peak and a bell shaped second reduction peak at 893 and $1050 \mathrm{~K}$, respectively. An interesting observation is that by introducing $\mathrm{Bi}$ and $\mathrm{Ni}$ promoters into the catalysts, the reduction patterns were significantly changed with more obvious reduction of $\mathrm{V}^{5+}$. Furthermore, these reduction peaks were at remarkably lower temperatures in the range of 820 to $860 \mathrm{~K}$. On the other hand, the second peak, which is associated with the reduction of $\mathrm{V}^{4+}$ species, appeared at lower temperatures (910-940 K) for all promoted VPO catalysts. A lower reduction temperature allows the VPO catalyst to operate with a lower energy consumption.

The amount of oxygen atoms removed from unpromoted and promoted VPO catalysts were calculated by a supplied software program, TPD/R/O 1100 version 2.3 . The number of oxygen species removed was given in $\mu \mathrm{mol} / \mathrm{g}$, and was converted to atom/g by multiplying with Avogrado constant (6.02 $\times 10^{23}$ ). As shown in Table 4, CatUnpromoted sample had the least amounts of oxygen atoms removed, and for the two peaks, these were $0.04 \times 10^{21}$ and $0.46 \times 10^{21}$ atom/g, respectively. The amount of oxygen atoms removed was increased by the

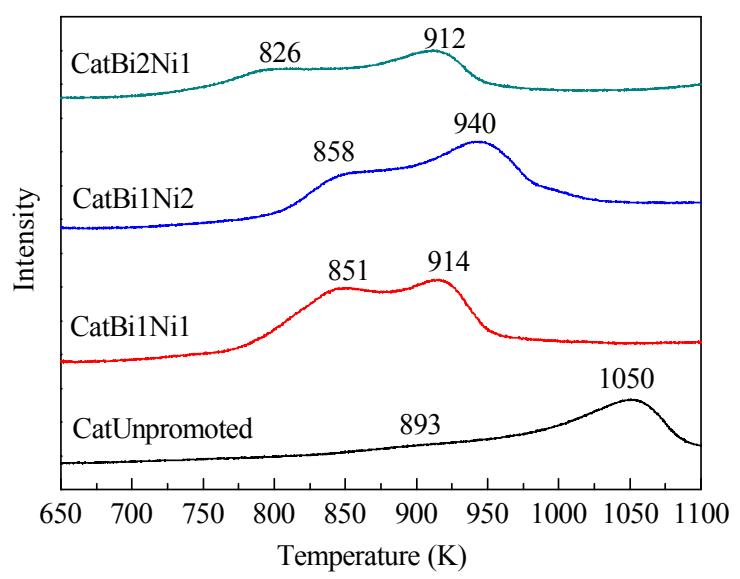

Fig. 4. $\mathrm{H}_{2}$-TPR profiles of unpromoted and promoted VPO catalysts. 
Table 4

Amount of oxygen atoms removed from unpromoted and promoted VPO catalysts by reduction in $\mathrm{H}_{2}$ /Ar.

\begin{tabular}{lccc}
\hline Catalyst & $\begin{array}{c}T_{\max } \\
(\mathrm{K})\end{array}$ & $\begin{array}{c}\text { Oxygen removed } \\
\left(10^{21} \text { atom } / \mathrm{g}\right)\end{array}$ & $\begin{array}{c}\mathrm{V}^{5+} / \mathrm{V}^{4+} \\
\text { ratio }\end{array}$ \\
\hline CatUnpromoted & 893 & 0.04 & 0.08 \\
& 1050 & 0.46 & \\
CatBi1Ni1 & 851 & 0.44 & 0.60 \\
& 914 & 0.73 & \\
CatBi1Ni2 & 858 & 0.36 & 0.52 \\
& 940 & 0.68 & \\
CatBi2Ni1 & 826 & 0.28 & 0.41 \\
& 912 & 0.67 & \\
\hline
\end{tabular}

addition of the Bi and Ni promoters. CatBi1Ni1 sample showed the largest amount of oxygen atoms removed, which was $0.44 \times$ $10^{21}$ atom/g sample for the low peak temperature (851 K) and $0.73 \times 10^{21}$ atom/g for the high peak temperature $(914 \mathrm{~K})$. The total amount of oxygen atoms removed from both peaks was also increased for promoted samples as compared to unpromoted one. Due to the large amount of reactive oxygen from $\mathrm{V}^{5+}$ removed from CatBi1Ni1, this catalyst gave the highest MA selectivity. In addition, the higher surface area may also contribute to the higher selectivity.

Thus, the addition of Bi and Ni successfully promoted VPO catalysts which consequently possessed more reactive and more labile oxygen species, which gave better reducibility compared to the unpromoted VPO catalyst. According to de Farias et al. [19], elements added in small amounts to give low promoter/vanadium atomic ratios act as an electronic promoter, and this was categorised as Type 2 promotion. CatBi1Ni1 was considered to be Type 2 . It consisted of $1 \% \mathrm{Bi}$ and $1 \% \mathrm{Ni}$ and showed the largest amount of oxygen atoms released in the two peaks. For the catalysts which contain $2 \%$ of either Bi or $\mathrm{Ni}$, the amount of oxygen atoms removed was slightly lower. In this Type 2 promotion, the cation or promoter was incorporated into the vanadyl pyrophosphate with $\mathrm{V}^{4+}$ in solid solution of the type ( $\left.(V O)_{1-x} \mathrm{M}_{x}\right)_{2} \mathrm{P}_{2} \mathrm{O}_{7}$ where $\mathrm{M}$ is a promoter cation. This may form defects in the structure which can act as new active sites or, alternatively, the metal cations may affect the redox properties of the catalyst surface.

In addition, there were more oxygen species removed at lower reduction temperature for the promoted VPO catalysts as compared with the unpromoted one because the former had an increased surface density of $\mathrm{V}=\mathrm{O}$ species on $(\mathrm{VO})_{2} \mathrm{P}_{2} \mathrm{O}_{7}$, which caused the $\mathrm{V}=\mathrm{O}$ bond at the adjacent site to become weaker. As a result, the specific activity increased, as reported by Takita et al. [30]. Ye et al. [20] found that the surface density of $V=0$ species on the (020) plane increased the exposure and disorder along the (020) plane. These $\mathrm{V}=0$ species are expected to act as active lattice oxygen, although several researchers suggested that adsorbed oxygen species were responsible for the reaction. Therefore, our observation suggested that Bi and Ni promoted VPO catalysts effectively improved the catalytic performance for the selective oxidation of $n$-butane towards MA.

The illustration of an ideal $(\mathrm{VO})_{2} \mathrm{P}_{2} \mathrm{O}_{7}$ plane and the metal promoter (M) substituted (020) plane is shown in Fig. 5 [30].

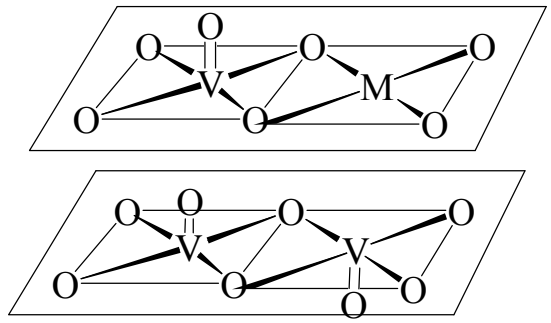

Fig. 5. Comparison between ideal and metal substituted (020) planes of $(\mathrm{VO})_{2} \mathrm{P}_{2} \mathrm{O}_{7}$.

\subsection{Catalytic oxidation of n-butane}

The effect of various $\mathrm{Bi} / \mathrm{Ni}$ ratios on the selectivity and activity of the catalysts are shown in Table 5. When VPO catalysts were promoted with $\mathrm{Bi}$ and $\mathrm{Ni}$, the catalytic performance was improved providing $n$-butane conversion of $51 \%$ and $52 \%$, while the MA selectivity was between $36 \%$ and $39 \%$ as compared to that over the unpromoted one (conversion 38\%, selectivity 28\%). These findings showed that the addition of promoters played an important role in enhancing the selectivity and activity of VPO catalysts. CatBi1Ni1 gave the highest $n$-butane conversion (52\%) and MA selectivity (39\%).

Figure 6 shows $n$-butane conversion as a function of amount of oxygen species removed associated with $\mathrm{V}^{4+}$. A good linear correlation coefficient $\left(R^{2}=0.84\right)$ between $n$-butane conversion and amount of oxygen species associated with $\mathrm{V}^{4+}$ (section 3.5) was observed. This proved that the catalyst activity was highly dependent on the presence of the $\mathrm{V}^{4+}$ phase. These results are in agreement with Taufiq-Yap et al. [27,29], who concluded that $\mathrm{V}^{4+}-\mathrm{O}^{-}$acted as the centre for the activation of $n$-butane. As reported earlier by Mars et al. [31], a large amount of surface

Table 5

Catalyst performance of unpromoted and promoted VPO catalysts.

\begin{tabular}{lcccc}
\hline \multirow{2}{*}{ Catalyst } & $\begin{array}{c}n \text {-Butane } \\
\text { conversion }(\%)\end{array}$ & \multicolumn{3}{c}{ Product selectivity (\%) } \\
\cline { 3 - 5 } CatUnpromoted & 38 & 28 & 71 & 1 \\
CatBi1Ni1 & 52 & 39 & 60 & 1 \\
CatBi1Ni2 & 52 & 37 & 62 & 1 \\
CatBi2Ni1 & 51 & 36 & 63 & 1 \\
\hline
\end{tabular}

Reaction conditions: $673 \mathrm{~K}, 1 \% n$-butane in air, GHSV $=2400 \mathrm{~h}^{-1}$.

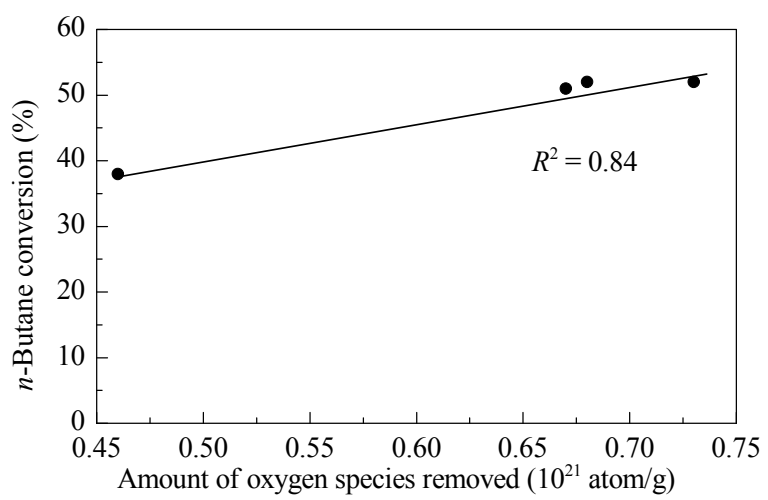

Fig. 6. $n$-Butane conversion as a function of the amount of oxygen species removed associated with $\mathrm{V}^{4+}$. 


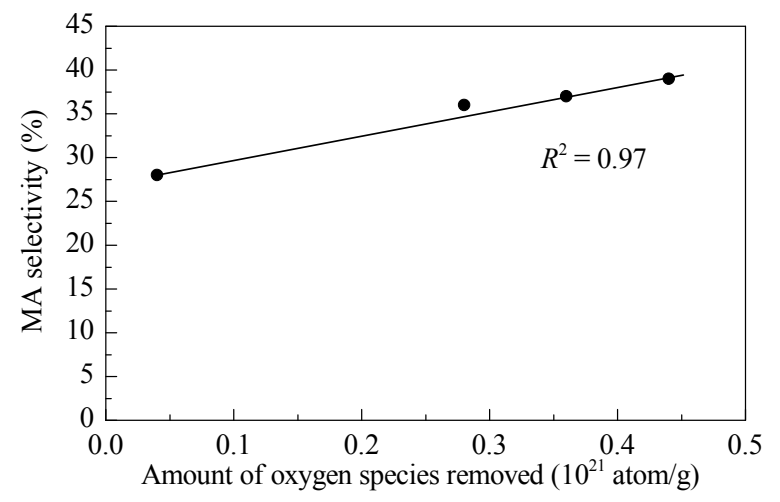

Fig. 7. MA selectivity as a function of amount of oxygen species removed associated with $\mathrm{V}^{5+}$.

lattice oxygen, which act as active species, also contribute to improving the catalyst activity. The results implied that this oxygen species was highly active for the selective oxidation of $n$-butane, and agreed with the electrical conductivity data obtained by Witko and co-workers [32]. CatBi1Ni1 gave the highest $n$-butane conversion (52\%) and removed $0.73 \times 10^{21}$ atom/g of oxygen species associated with $\mathrm{V}^{4+}$.

Figure 7 shows the selectivity to MA as a function of the amount of oxygen species removed associated with $\mathrm{V}^{5+}$. The linear graph shows a good correlation with a high correlation coefficient $\left(R^{2}=0.97\right)$ which indicated that these oxygen species $\left(\mathrm{O}^{2-}\right)$ amounts were directly proportional to the selectivity of MA $[28,29,33]$. It has been shown that the $\mathrm{V}^{5+}$ species play an essential role in hydrogen abstraction from $n$-butane [34]. The presence of $\mathrm{V}^{5+}$ surface species would favour selective oxidation by preventing or limiting the over-oxidation of adsorbed MA molecule [35]. All the Bi and Ni promoted VPO catalysts gave larger amounts of the active oxygen species, $\mathrm{O}^{2-}$ associated with $\mathrm{V}^{5+}$, which contributed to better MA selectivity. It was proved that the promoted VPO catalysts showed higher catalytic performance. CatBi1Ni1 gave the highest MA selectivity $(39 \%)$ and it removed $0.44 \times 10^{21}$ atom/g of oxygen species associated with $\mathrm{V}^{5+}$.

The principle role of the promoters is to enable the formation of smaller crystallite size in the (020) plane direction. According to Ye et al. [20], a smaller crystallite size with the broadening of (020) plane and disorder along the (020) plane were present after modification by the addition of promoters. It has been widely accepted that the $(020)$ plane is active for the selective oxidation of $n$-butane, which leads to the expectation

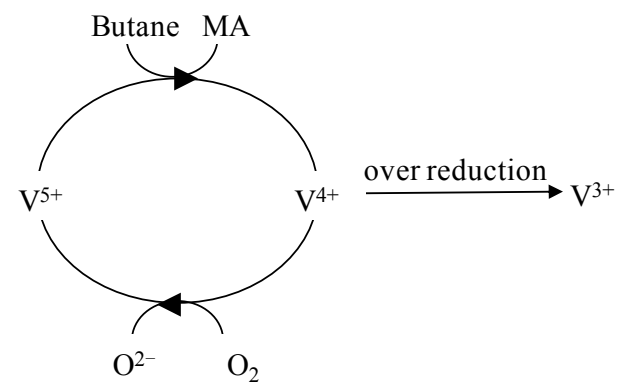

Fig. 8. Changes of vanadium valence during $n$-butane oxidation over VPO catalyst. that a high exposure of the (020) plane will result in higher activity and selectivity.

During the reaction of $n$-butane to MA, a surface redox cycle takes place between $\mathrm{V}^{5+}$ and $\mathrm{V}^{4+}$, i.e. surface $\mathrm{V}^{5+}$ is first reduced by butane to $\mathrm{V}^{4+}$, then it is quickly oxidized to $\mathrm{V}^{5+}$ with either adsorbed or gas phase oxygen [36]. If over-oxidation occurs, $\mathrm{V}^{4+}$ is reduced to $\mathrm{V}^{3+}$ as shown in Fig. 8.

\section{Conclusions}

Among the three different $\mathrm{Bi}$ and Ni promoted VPO catalysts for $n$-butane oxidation to MA, CatBi1Ni1 was the most active catalyst with a conversion of $52 \%$ and MA selectivity of $39 \%$. All the $\mathrm{Bi}$ and $\mathrm{Ni}$ promoted catalysts have smaller crystallite size and higher specific surface areas as compared to the unpromoted one. The reduction behaviour over the promoted catalysts were investigated to discover the role of oxygen species in this selective oxidation reaction. There was a good correlation between the amount of oxygen species associated with $\mathrm{V}^{4+}$ and $n$-butane conversion. All the promoted VPO catalysts with a larger amount of active oxygen species, $\mathrm{O}^{-}$associated with $\mathrm{V}^{4+}$, gave higher catalytic activity. The excess amount of oxygen species $\left(\mathrm{O}^{2-}\right)$, which is associated with $\mathrm{V}^{5+}$, significantly increased MA selectivity.

\section{References}

[1] Centi G, Gleaves J T, Golinelli G, Trifiro F. Stud Surf Sci Catal, 1992, 72: 231

[2] Trivedi B C, Culbertson B M. Maleic Anhydride. New York: Plenum Press, 1982.871

[3] Hutchings G J. Catal Today, 1993, 16: 139

[4] Bartley J K, Lopez-Sanchez J A, Hutchings G J. Catal Today, 2003, 81: 197

[5] Hutchings G J. Appl Catal, 1991, 72: 1

[6] Hutchings G J, Higgins R.J Catal, 1996, 162: 153

[7] Centi G, Cavani F, Trifiro F. Selective Oxidation by Heterogeneous Catalysis. New York: Kluwer Academic Plenum Publisher, 2001. 158

[8] Edwards R C. US Patent 4515904 A. 1985

[9] Bortinger A. US Patent 5885919 A. 1999

[10] Sookraj S H, Engelbrecht D. Catal Today, 1999, 49: 161

[11] Taufiq-Yap Y H, Tan K P, Waugh K C, Hussein M Z, Ramli I, Abdul Rahman M B. Catal Lett, 2003, 89: 87

[12] Taufiq-Yap Y H, Kamiya Y, Tan K P. J Nat Gas Chem, 2006, 15: 297

[13] Taufiq-Yap Y H, Abdul Ghani A A. Chin J Catal (催化学报), 2007, 28: 1037

[14] Taufiq-Yap Y H, Nurul Suziana N M, Hussein M Z. Catal Lett, 2011, 141: 136

[15] Haber J, Zazhigalov V A, Stoch J, Bogutskaya L V, Batcherikova I V. Catal Today, 1997, 33: 39

[16] Klug H P, Alexander L E. X-Ray Diffraction Procedures for Polycrystalline and Amorphous. New York: John Wiley \& Sons, 1974. 618

[17] Kesteman E, Merzouki M, Taouk B, Bordes E, Contractor R. Stud Surf Sci Catal, 1995, 91: 707

[18] Igarashi H, Tsuji K, Okuhara T, Misono M. J Phys Chem, 1993, 97: 7065

[19] de Farias A M D, Gonzalez W d A, de Oliveira P G P, Eon J G, Herrmann J M, Aouine M, Loridant S, Volta J C. J Catal, 2002, 208 : 


\section{Graphical Abstract}

Chin. J. Catal., 2014, 35: 270-276 doi: 10.1016/S1872-2067(12)60749-9

Effects of $\mathrm{Bi}$ and $\mathrm{Ni}$ on the properties of a vanadium phosphorus oxide catalyst

Yun Hin Taufiq-Yap*, Choon Seon Yuen, Nawi @ Mohamed Nurul Suziana, Ramli Irmawati Universiti Putra Malaysia, Malaysia

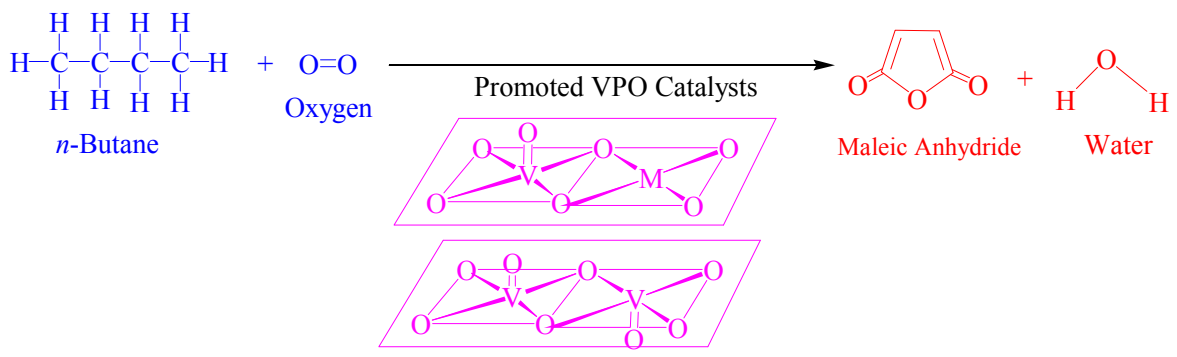

Note: Promoter denoted as M

Bi and Ni promoters in a VPO catalyst improved its catalytic properties in selective oxidation of $n$-butane to maleic anhydride.

\section{8}

[20] Ye D, Satsuma A, Hattori A, Hattori T, Murakami T. Catal Today, 1993, 16: 113

[21] Hutchings G J.J Matter Chem, 2004, 14: 3385

[22] Centi G. Catal Today, 1993, 16: 5

[23] Centi G, Manenti I, Riva A,Trifiro F. Appl Catal, 1984, 9: 177

[24] Poli G, Resta I, Ruggeri O, Trifiro F. Appl Catal, 1981, 1: 395

[25] Centi G, Trifiro F, Ebner J R, Franchetti V M. Chem Rev, 1988, 88: 55

[26] Kiely C J, Sajip S, Ellison I J, Sananes M T, Hutchings, G J, Volta J C. Catal Lett, 1995, 33: 357

[27] Taufiq-Yap Y H, Goh C K, Hutchings G J, Dummer N, Bartley J K. Catal Lett, 2009, 130: 327
[28] Abon M, Herrmann J M, Volta J C. Catal Today, 2001, 71: 121

[29] Taufiq-Yap Y H, Goh C K, Hutchings G J, Dummer N, Bartley J K. J Mol Catal A, 2006, 260: 24

[30] Takita Y, Tanaka K, Ichimaru S, Mizihara Y, Abe Y, Ishihara T. Appl Catal A, 1993, 103: 281

[31] Mars P, van Krevelen D W. Chem Eng Sci, 1954, 3: 41

[32] Witko M, Tokarz R, Haber J, Hermann K. J Mol Catal A, 2001, 166: 59

[33] Volta J C. Top Catal, 2001, 15: 121

[34] Lin M M. Appl Catal A, 2003, 250: 287

[35] Joly J P, Mehier C, Bere K E, Abon M. Appl Catal A, 1998, 169: 55

[36] Wang D X, Kung H H, Barteau M A. Appl Catal A, 2000, 201: 203 